\title{
A Comparative Study on Movement Feature in Different Directions for Micro-Expression Recognition
}

\author{
Jinsheng $\mathrm{Wei}^{1}$, Guanming Lu ${ }^{1, *}$, Jingjie Yan $^{1}$ \\ Nanjing University of Posts and Telecommunications, Nanjing (210003), China
}

\begin{abstract}
Micro-expression can reflect people's real emotions. Recognizing micro-expressions is difficult because they are small motions and have a short duration. As the research is deepening into micro-expression recognition, many effective features and methods have been proposed. To determine which direction of movement feature is easier for distinguishing micro-expressions, this paper selects 18 directions (including three types of horizontal, vertical and oblique movements) and proposes a new low-dimensional feature called the Histogram of Single Direction Gradient (HSDG) to study this topic. In this paper, HSDG in every direction is concatenated with LBP-TOP to obtain the LBP with Single Direction Gradient (LBP-SDG) and analyze which direction of movement feature is more discriminative for micro-expression recognition. As with some existing work, Euler Video Magnification (EVM) is employed as a preprocessing step. The experiments on the CASME II and SMIC-HS databases summarize the effective and optimal directions and demonstrate that HSDG in an optimal direction is discriminative, and the corresponding LBP-SDG achieves state-of-the-art performance using EVM.
\end{abstract}

Keywords: Micro-Expression Recognition, Movement Feature, Histogram of Single Direction Gradient, Effective Direction

\section{Introduction}

Facial expression can be divided into macroexpression and micro-expression. Macro-expression is an expression which is observed on the face directly, but when people want to hide their real emotions, we cannot infer their real emotions from macro-expression. When people try to hide their real emotions, micro-expression can represent their real emotions. Although people's real emotions can be inferred from micro-expression, micro-expression cannot be recognized as easily as macro-expression because it is momentary and minor.

Micro-expressions have great research significance and have been studied in the field of psychology for many years 1, 2, 3, 4, 5. Micro-expression has been proven to be effective in reflecting people's real emotions, and recognizing micro-expression

\footnotetext{
* Corresponding author

Email addresses: 2018010217@njupt.edu.cn (Jinsheng Wei), lugm@njupt.edu.cn (Guanming Lu), yanjingjie@njupt.edu.cn (Jingjie Yan)
}

is valuable for many applications, including lie detection [6, 7], medical diagnosis [5], public safety [8] and so on 9,10 . Ekman et al. developed the MicroExpression Training Tool (METT) 11 to train ordinary people to recognize micro-expressions in seven categories. As micro-expression has minor movements and a momentary duration, recognizing it is artificially difficult. In [12, it was shown that undergraduate students who receive the help of METT can only achieve about an accuracy of $40 \%$ in detecting micro-expression. Fortunately, the development of computer vision and pattern recognition has promoted research into automatic microexpression recognition, and recent research has shown that automatic micro-expression recognition can achieve an excellent recognition rate(from here on, "micro-expression recognition" below means "automatic micro-expression recognition").

To study micro-expression recognition more conveniently, many teams have collected microexpression video databases that are used to verify the effectiveness of their proposed method. 
Micro-expression databases mainly include spontaneous and non-spontaneous types, and spontaneous databases are more difficult to collect and more realistic than non-spontaneous databases. At present, commonly used spontaneous databases include CASME II[13], SMIC 14], SAMM 15] and so on. The recognition rate for these databases has been improved continuously, but micro-expression recognition still faces many challenges, and more effective and robust methods are still needed to promote micro-expression recognition. In this paper, an effective feature is proposed to study movement feature in different directions and improve the recognition rate, and the proposed feature obtains state-of-the-art performance after being concatenated with LBP-TOP.

\section{Related Works}

This section introduces the related works that have been explored in the field of micro-expression recognition. The recognition of micro-expressions mainly includes three parts: preprocessing, feature extraction and classification. Since the focus of this paper is feature extraction, the following section introduces preprocessing and classification briefly and presents feature extraction in more detail.

In the early stage, some simple explorations and attempts were presented regarding preprocessing and classifiers, but the two parts are relatively fixed. Many databases use video clips where faces have been detected, intercepted and aligned. Eulerian Video Magnification(EVM) [16] and Time Interpolation Model(TIM) 17] also are effective preprocessing methods, which have been used widely. The effectiveness of EVM and TIM has been proven in a great deal of work, but they are treated separately. Recently, Peng et al. 18 combined TIM and EVM to eliminate the side effects caused by the intermediate process and to obtain a state of the art recognition rate. For classifiers, to date, AdaBoost 19, Softmax 20, KNN 21] and so on [22, 23] have been selected, but the most common and effective classifier still is the Support Vector Machine (SVM).

Feature extraction is a key step in microexpression recognition, and it has always been a research focus. A large number of features have been proposed, such as LBP-TOP, LBP-IP, HIGO, etc. These features can be roughly divided into LBP-based, optical flow-based, gradient-based and deep learning-based methods.

\section{LBP based features}

LBP descriptor was proposed to extract texture feature from two-dimensional images by Ojala 24]. Zhao et al. 25] extended LBP from two-dimensional images to three-dimensional videos and obtained LBP-TOP to describe dynamic texture of facial expression video. LBP-TOP is very effective for video analysis, and the LBP-TOP based features have been a research hotspot in micro-expression recognition. A large number of teams have made innovative work. Inspired by the concept of LBPTOP, Wang et al. proposed more compact LBP$\mathrm{SIP}$ [26] and LBP-MOP 27]. LBP-SIP removes redundant points and only calculates the LBP value of six points, and LBP-MOP descriptor does not extract the features from all frames but extracts the features from the average plane. Huang et al. 28. proposed SpatioTemporal Completed Local Quantization Patterns (STCLQP) and used effective vector quantization and codebook selection to process extracted sign, magnitude and orientation components. After that, they put forward Spatiotemporal Local Binary Pattern with Integral Projection (STLBP-IP 29]) based on difference image; they calculated the difference images and then extracted LBP features from the integral projection map of the difference images. Huang 30] and Zong[22] proposed Discriminative and Hierarchical STLBP-IP respectively to enhance the STLBP-IP feature. Wang et al.31 introduced EVM into micro-expression recognition as a preprocessing step and then extracted LBP-TOP to achieve a satisfactory recognition rate. However, their work does not give the recognition rate under the LOSO cross-validation and only tested their method on the CASME II database.

\section{optical flow based features}

Optical Flow (OF) technology was first proposed by Horn et al. 32 and proven to be effective for micro-expression recognition by several studies. Early, Liong et al. 33] employed Optical Strain (OS) feature to recognize micro-expressions, and in the work 34, the LBP-TOP was weighted by the temporal mean-pooled OS map in every region; after that, they proposed a Bi-Weighted Oriented Optical Flow (BI-WOOF) 35 that locally and globally weights HOOF features. To eliminate the influence of noise and illumination changes, Xu et al. 36. proposed the Facial Dynamics Map (FDM) feature to select the principal direction from the optical 
flow map. Using the ROI-based OF feature, Liu et al. 37. presented Main Directional Mean Optical flow (MDMO) to choose the main direction in every ROI. Recently, they 38 employed sparse representation technology to enhance the feature representation of MDMO and achieve a promising recognition rate. Furthermore, some works combine optical flow and histogram. Zhang et al. 23] aggregated the Histogram of the Oriented Optical Flow (HOOF) with LBP-TOP features, and inspired by the idea of the fuzzy color histogram, Happy et al. 39 proposed Fuzzy Histogram of Optical Flow Orientation (FHOFO) that is robust to the variation of expression intensities.

\section{gradient based features}

The gradient descriptor can extract the movement feature. Histograms of Oriented Gradients (HOG) is an effective gradient feature, and HOGTOP can be effectively applied to micro-expression recognition after extending HOG to three orthogonal planes. HOG-TOP was first employed by Polikovsky et al. 40 for micro-expression recognition. In their work, two gradient operators were used to calculate the vertical and horizontal gradients of each pixel in every region of interest (ROI) from three orthogonal planes; then, the gradient direction and gradient magnitude were calculated according to the horizontal and vertical gradients, and the gradient direction weighted by the gradient magnitude was quantified; finally, histogram operation was employed to process these quantized directions. Recently, the histogram of image gradient orientation (HIGO) proposed by Li et al. 41] does not use the gradient magnitude to weight the gradient direction, and their work employed EVM as a preprocessing method and then achieved an excellent recognition rate.

\section{deep learning based features}

Recently, deep learning method has been applied in many fields, and Convolutional Neural Network $(\mathrm{CNN})$ is an effective method in the field of image understanding. Because of the limited sample size, CNN model is difficult to be trained for micro-expression recognition. To solve this problem, the pre-trained VGG model and data enhancement technology were employed in the work 42, and then the model was fine-tuned to recognize micro-expression using the micro-expression apex frame. Kim et al. 20] adopted CNN and the
Long Short-Term Memory (LSTM) Recurrent Neural Networks to extract spatial features of five states and temporal features respectively. The work 43 . directly use CNN to extract the spatial features of each frame and input these features into LSTM. 3D Convolution Neural Network is a deep learning method for video processing. Li et al. 44 tried this method in the micro-expression recognition; and in their work, the optical flow maps (horizontal and vertical) and gray-scale frames are gathered and then input into a designed 3DCNN model. Also, the optical flow maps (horizontal and vertical) and the optical strain image are stacked together and input into CNN in work 45. The above works promoted the application of deep learning in micro-expression recognition, but their recognition rates are not outstanding compared with traditional methods. By incorporating Accretion Layers (AL) in the network, Verma et al. 46] proposed a Lateral Accretive Hybrid Network (LEARNet) that refines the salient expression features in accretive manner. Their method achieves a excellent recognition rate, but their experiment don't adopts the mainstream cross-validation method. Furthermore, Khor et al. 47. proposed a lightweight dualstream shallow network that has the form of a pair of truncated CNNs with heterogeneous input features, and this method obtains state of the art performance on CASME II database. Recently, using apex frame in micro-expression video, Song et al. 48. designed a dynamic-temporal stream, staticspatial stream, and local-spatial stream module for the TSCNN that respectively attempt to learn and integrate temporal, entire facial region, and facial local region cues to recognize micro-expressions, and TSCNN achieves the promising recognition. Also, the traditional methods separate feature extraction and classification, while the deep learning methods merge feature extraction and classification and get one model to extract features and classify micro-expressions.

The optical flow or gradient-based descriptors can extract movement features, but which direction of movement is most conducive for distinguishing micro-expression is still unclear? Extracting the movement feature can be divided into two parts: passive and active. Here, 'passive' means that the extracted movement feature is based on the current video data (for example, in a video clip, if the direction of muscle movement is mainly direction A, the extracted movement feature describes the movement in direction A), while 'active' means ex- 
tracting the movement feature in a certain direction for all video clips (for example, in all video clips, the movement feature is extracted in the specified direction B.). To study the movement feature in different directions, the 'active' method is more suitable. In existing works, both optical flow and gradientbased descriptors adopted the 'passive' method to extract movement features, so these works cannot be used directly. Besides, according to the definition of optical flow, the optical flow based descriptors cannot be employed to extract movement feature in a specific direction. Thus, we innovated the gradient-based HOG descriptor to meet this demand.

For the studied aim, this paper proposes a new low-dimensional feature called the Histogram of Single Direction Gradient (HSDG) that actively extracts movement feature in a certain direction. The innovation of HSDG is that it simplifies HOG and extracts the movement feature actively. Specifically, on one hand, HSDG only extracts the gradient value in a single direction to ensure that the movement feature in a certain direction are extracted; on the other hand, it does not calculate the gradient direction based on these gradient values, while it directly quantizes these gradient values to reduce the feature dimension. Furthermore, considering that LBP-TOP provides effective appearance texture information, and HSDG cannot provide comprehensive feature information, LBP-TOP, as a basic feature, is concatenated with HSDG to obtain LBP with a Single Direction Gradient(LBPSDG). The contributions in this paper can be summarized as follows:

1) In this paper, we study movement feature in different directions and summarize the directions in which movement feature is more useful to distinguish micro-expressions. The experimental results show that the effective direction tends to be the same under different magnification coefficients of EVM.

2) For the studied aim, a new feature HSDG is proposed. HSDG descriptor can actively extract movement feature in a specific direction. LBP-SDG concatenates HSDG with LBP-TOP and achieves state-of-the-art performance after using EVM.

3) HSDG is a low-dimensional feature, and the comparative experiments are used. The results demonstrate that HSDG in the optimal direc- tion is a discriminative feature and can provide effective information. Furthermore, HSDG can be taken as a supplementary feature to improve the performance of basic features.

\section{The Proposed Method}

This section introduces our proposed method for the studied aim. The proposed HSDG feature provides effective feature information and achieves a competitive recognition rate after concatenating with LBP-TOP. The proposed method mainly involves preprocessing (EVM, TIM), feature extraction (LBP-TOP, and HSDG). These are described below.

\subsection{Pre-processing}

In our work, two effective preprocessing methods (EVM and TIM) were employed, and we introduce the two technologies briefly below.

The difficulties of recognizing micro-expression lie in the fact that micro-expressions are tiny facial movements and have a short occurrence time. Fortunately, EVM can enlarge the muscle movement in the video and has been used widely in microexpression recognition, so our work also employed this technology. In EVM, the magnification factor Alpha is a key parameter and its value cannot be arbitrarily selected or infinitely large. The optimal Alpha value is different for different databases, so it was fine-selected in the experiment. For detail about EVM, refer to [16]. Collecting spontaneous micro-expression databases is difficult and the number of collected video frames is uneven. TIM as a normalization method can unify the frames number to solve the problem about the uneven number of frames. TIM was originally proposed for a lipreading system and achieved a very good recognition rate. For detail about TIM, refer to 17 .

In our work, the cropped databases that have operated preprocessing such as size normalization, face interception, and face alignment were used directly, and apart from EVM and TIM, no other preprocessing methods are employed.

\subsection{Feature Extraction}

Feature extraction is the key step of microexpression recognition and is also the focus of this paper. HSDG is proposed for the studied aim, but the feature information provided by HSDG is incomprehensive. Therefore, HSDG is concatenated 
with LBP-TOP to obtain LBP-SDG. LBP-TOP and HSDG are introduced in this section.

\subsection{1. $L B P-T O P$}

A large amount of previous work in different fields shows that LBP-TOP is very effective in video analysis. LBP-TOP can extract texture features from three planes: XY, XT, and YT. The appearance texture features are extracted from the XY plane, while the dynamic texture features are extracted from the other two planes. As LBP is the basis of LBP-TOP, this section first introduces LBP for ease of understanding, and then presents LBP-TOP.

\section{$L B P$}

LBP extracts texture features from twodimensional images. Suppose $I$ is a twodimensional image, and a point $P_{I}=\left(X_{I}, Y_{I}\right)$ in $I$ is taken as the center point; also, $R_{X I}$ and $R_{Y I}$ are the radii on the horizontal and vertical directions, respectively, and $N_{I}$ points around $P_{I}$ are selected to calculate the LBP value. First, the coordinates of the $N_{I}$ points are confirmed, and the coordinates of the i-th point $D_{i}$ are:

$$
\begin{array}{r}
D_{i}=D\left(X_{I}, Y_{I}, R_{X I}, R_{Y I}, N_{I}, i\right)=\left(X_{I}+R_{X I}\right. \\
\left.* \sin \left(2 \pi i / N_{I}\right), Y_{I}+R_{Y I} * \cos \left(2 \pi i / N_{I}\right)\right) \\
i=0,1 \ldots N_{I}-1
\end{array}
$$

These calculated coordinate points form an ordered point set $D_{I}$. Based on $D_{I}$, the LBP value of the point $P_{I}$ is calculated:

$$
\operatorname{LBP}\left(P_{I}, D_{I}\right)=\sum_{i=0}^{N_{I}-1} \varepsilon\left(g\left(D_{i}\right)-g\left(P_{I}\right)\right) * 2^{i}
$$

Where $g(x)$ represents the pixel value at point $x$; i represents the index of points in $D_{I}$; and the $\varepsilon(*)$ function is defined as follows:

$$
\varepsilon(x)= \begin{cases}0 & x<0 \\ 1 & x \geq 0\end{cases}
$$

Finally, the LBP values of all points on $I$ are calculated, and the histogram of these LBP values is the LBP feature.

\section{$L B P-T O P$}

After presenting the concept of LBP, LBP-TOP is easy to explain. LBP-TOP concatenates these
LBP features from the three planes. A threedimensional video has three coordinate axes (horizontal X, vertical $\mathrm{Y}$, time $\mathrm{T}$ ), and these three coordinate axes can form three planes (XY, XT, YT). Suppose $V$ is a three-dimensional video, and a point $P_{V}=\left(X_{V}, Y_{V}, T_{V}\right)$ in $V$ is taken as the center point; also, $R_{X V}, R_{Y V}$, and $R_{T V}$ are the radii in the horizontal, vertical and time directions, respectively, and $N_{X Y V}, N_{X T V}$ and $N_{Y T V}$ points around $P_{V}$ are selected to calculate the LBP values from the plane XY, XT, and YT, respectively. First, the coordinates of the i-th point $\left(D_{X Y i}, D_{X T i}\right.$, and $\left.D_{Y T i}\right)$ on the three planes are as follows:

$$
D_{X Y i}=D\left(X_{V}, Y_{V},+R_{X V},-R_{Y V}, N_{X Y V}, i\right)
$$

$$
\begin{gathered}
D_{X T i}=D\left(X_{V}, T_{V},+R_{X V},+R_{T V}, N_{X T V}, i\right) \\
D_{Y T i}=D\left(Y_{V}, T_{V},-R_{Y V},+R_{T V}, N_{Y T V}, i\right)
\end{gathered}
$$

where function $D(*, *, *, *, *)$ mentioned in Equation 1. $X Y i=0,1, \ldots, N_{X Y V}-1 ; \quad X T i=$ $0,1, \ldots, N_{X T V}-1$; and $Y T i=0,1, \ldots, N_{Y T V}-1$.

All $D_{X Y i}, D_{X T i}$, and $D_{Y T i}$ over all i form three ordered point sets, $D_{X Y}, D_{X T}$, and $D_{Y T}$, respectively. Next, the LBP values $\left(L B P_{X Y}, L B P_{X T}\right.$, and $L B P_{Y T}$ ) of point $P_{V}$ on the three planes are determined by the following equation:

$$
\begin{aligned}
& L B P_{X Y}\left(P_{V}\right)=\operatorname{LBP}\left(P_{V}, D_{X Y}\right) \\
& L B P_{X T}\left(P_{V}\right)=L B P\left(P_{V}, D_{X T}\right) \\
& L B P_{Y T}\left(P_{V}\right)=L B P\left(P_{V}, D_{Y T}\right)
\end{aligned}
$$

Where function $\operatorname{LBP}(*, *)$ mentioned in Equation 2

Finally, these LBP features from three planes are calculated and concatenated to get LBP-TOP.

\subsection{2. $H S D G$}

The studied aim in this paper is to determine which directions of movement feature are most effective, so we need to actively extract movement features in a single direction. Considering the gradient feature can extract the movement feature as described by [4], the proposed HSDG feature employs the gradient method and extracts gradient features in a single direction. Besides, the proposed feature is inspired by $\mathrm{HOG}$ features to calculate gradient values in a single direction and then the histogram of these gradient values. Furthermore, 


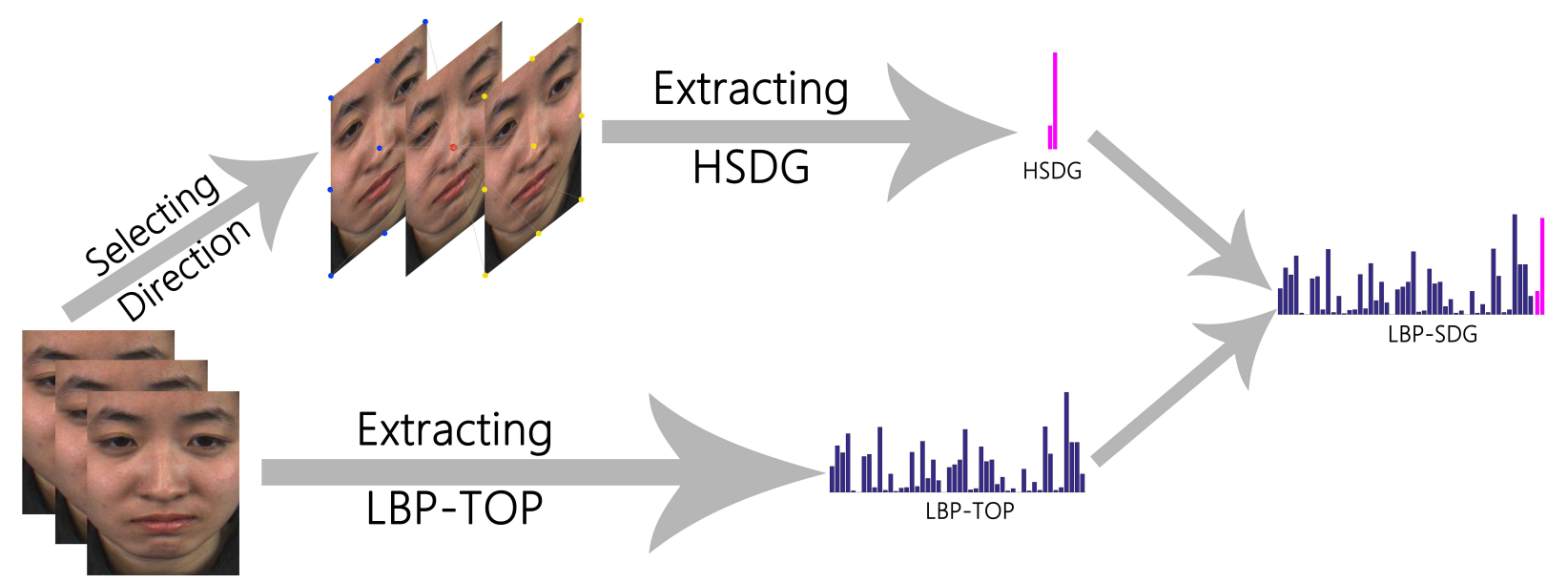

Figure 1: The framework of extracting LBP-SDG. LBP-TOP and HSDG features are extracted from the micro-expression video clips before these two features are concatenated to obtain LBP-SDG.

before calculating the histogram, these gradient values are quantified into several values to reduce the feature dimension.

The differences between HOG/HIGO and HSDG are as follows: first, HOG/HIGO calculates the gradient direction according to the calculated horizontal and vertical gradient values, while HSDG calculates the gradient value in only a certain direction. Second, HOG/HIGO quantifies the gradient direction into several directions, while HSDG quantizes the calculated gradient value into several values. Third, overall, HOG/HIGO calculates the gradient direction passively, while HSDG calculates the gradient values in a single direction actively. The specific details of HOG/HIGO can be found in [50] and [41, and the method of calculating HSDG is as follows.

\section{Directions}

Before introducing HSDG, the 18 directions were selected for testing. In $V$, we select $P_{V}=$ $\left(X_{V}, Y_{V}, T_{V}\right)$ as the center point, and $R_{X}, R_{Y}$ and $R_{T}$ are the radii in the horizontal, vertical and time directions, respectively. The point (i) is selected in $V$, and $\mathrm{i}$ is from 1 to 18 . As the extracted feature is used to describe movement information, $R_{T}$ must not be equal to 0 ; that is, the points should be selected from the previous or subsequent frames. As shown in Figure 2 six points, four points, and eight points are selected on the horizontal (XT), vertical (YT), and oblique planes, respectively; for example, point (11) belongs to the horizontal plane.

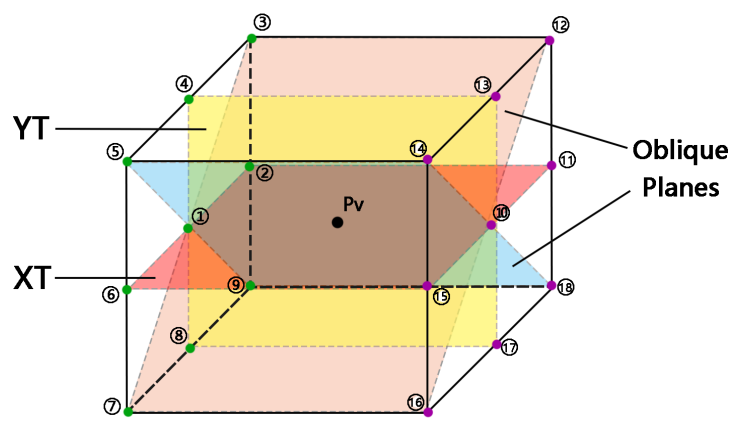

Figure 2: The selected 18 directions. $P_{V}$ represents the center point. The directions (1), (2), (6), (10), (11) and (15) lie the horizontal plane (XT), the directions (4), 8), (13) and (17) lie the vertical plane (YT), and the other directions lie the oblique planes.

Then, based on these points, $P_{V}$ (i) is selected as the tested direction. Since the directions are numbered as the same order of point, and (i) is used directly to represent the direction in the subsequent 
text. The selected 18 points form a point set D:

$$
\begin{aligned}
D=\{ & \left(X_{V}+0, Y_{V}+0, T_{V}-R_{T}\right), \\
& \left(X_{V}-R_{X}, Y_{V}+0, T_{V}-R_{T}\right), \\
& \left(X_{V}-R_{X}, Y_{V}+R_{Y}, T_{V}-R_{T}\right), \\
& \left(X_{V}+0, Y_{V}+R_{Y}, T_{V}-R_{T}\right), \\
& \left(X_{V}+R_{X}, Y_{V}+R_{Y}, T_{V}-R_{T}\right), \\
& \left(X_{V}+R_{X}, Y_{V}+0, T_{V}-R_{T}\right), \\
& \left(X_{V}+R_{X}, Y_{V}-R_{Y}, T_{V}-R_{T}\right), \\
& \left(X_{V}+0, Y_{V}-R_{Y}, T_{V}-R_{T}\right), \\
& \left(X_{V}-R_{X}, Y_{V}-R_{Y}, T_{V}-R_{T}\right), \\
& \left(X_{V}+0, Y_{V}+0, T_{V}+R_{T}\right), \\
& \left(X_{V}-R_{X}, Y_{V}+0, T_{V}+R_{T}\right), \\
& \left(X_{V}-R_{X}, Y_{V}+R_{Y}, T_{V}+R_{T}\right), \\
& \left(X_{V}+0, Y_{V}+R_{Y}, T_{V}+R_{T}\right), \\
& \left(X_{V}+R_{X}, Y_{V}+R_{Y}, T_{V}+R_{T}\right), \\
& \left(X_{V}+R_{X}, Y_{V}+0, T_{V}+R_{T}\right), \\
& \left(X_{V}+R_{X}, Y_{V}-R_{Y}, T_{V}+R_{T}\right), \\
& \left(X_{V}+0, Y_{V}-R_{Y}, T_{V}+R_{T}\right), \\
& \left.\left(X_{V}-R_{X}, Y_{V}-R_{Y}, T_{V}+R_{T}\right)\right\}
\end{aligned}
$$

Theoretically, the movement features between directions (1) - (9) and directions (10) - (18) should be consistent. For example, the movement features in direction (7) and direction (12) should be similar, since the two directions are on the same line. However, since HSDG and LBP-TOP adopt the same method of block division for consistency and the efficiency of the extracted feature, the points in the front and rear $R_{T V}$ frames of a video cannot be taken as the center point. Thus, the points in the rear $R_{T V}$ frames cannot be used when extracting HSDG in directions (1) - (9), while the points in the former $R_{T V}$ frame cannot be used for extracting HSDG in directions (10) - (18). Therefore, the movement features in directions (1) - (9) and directions (10) - (18) are different and are tested separately.

\section{Extraction Detail}

We pick a direction, and the HSDG features in the direction can then be extracted. First, we select a point $\mathrm{P}$ from $\mathrm{D}$; then, the gradient in the direction $\overrightarrow{P_{V} P}$ is:

$$
G\left(\overrightarrow{P_{V} P}\right)=g(P)-g\left(P_{V}\right)
$$

As the pixel value ranges from 0 to 255 , the gradient value ranges from -255 to 255 . Considering that the histogram operation on all gradient values(511 types) will generate a huge feature dimension, all gradient values are quantized to $\mathrm{N}$ values. All gradient values are quantified by the following equation:

$$
q(x)= \begin{cases}0 & -255 \leq x<f\left(-255+\frac{511 * 1}{N}\right) \\ 1 & f\left(-255+\frac{511 * 1}{N}\right) \leq x<f\left(-255+\frac{511 * 2}{N}\right) \\ \cdots & \ldots \\ N-1 & f\left(-255+\frac{511 *(N-1)}{N}\right) \leq x \leq 255\end{cases}
$$

where $\mathrm{f}(\mathrm{x})$ is Rounddown function, and the input $\mathrm{x}$ of $\mathrm{q}(\mathrm{x})$ is the gradient value.

After the quantized gradient values at all points are calculated, the histogram of these values is the HSDG features.

When a facial muscle movement appears in an area, it will cause corresponding facial movement variations in the area and its surrounding areas. These variations are reflected in the video data as the variations of the pixel values. The proposed HSDG descriptor can extract movement features in a certain direction, and HSDG feature can express the variations of the pixel values in a certain direction. 18 representative directions were selected and the planes where these directions lie include horizontal, vertical, and oblique planes. Among these tested directions, the effective direction means that the movement features (the variations of the pixel values) in this direction are discriminative for distinguishing micro-expressions. In other words, facial movement variations in this direction are good for recognizing micro-expressions. It is noted that directions (1) and (10) are the directions along the time axis, and HSDG features in the two directions express the variations of the pixel values in the same pixel at different times.

\subsection{3. $L B P-S D G$}

HSDG descriptor can meet the studied aim in this paper, but the movement feature extracted by it is only in a single direction and provides limited feature information. LBP-TOP descriptor can extract appearance texture and dynamic texture features and provides relatively sufficient texture feature information. Thus, HSDG is concatenated with LBP-TOP to obtain LBP-SDG for studying the movement features in different directions. Taking the recognition rate of $\mathrm{LBP}-\mathrm{TOP}$ as a reference, when the HSDG feature in a certain direction is added, if the recognition rate of LBP-TOP is im- 
proved, the movement feature in this direction can provide effective information. The entire feature extraction process is shown in Figure 1 .

\section{Experiments}

A large number of experimental results and the analysis are shown in this section. Our experiments tested LBP-SDG in 18 directions to summarize the directions in which movement feature is effective for recognizing micro-expressions. If the recognition rate of LBP-SDG in a certain direction is higher than that of LBP-TOP, the movement feature provided by the corresponding HSDG is effective; that is, the movement feature in this direction is effective, and vice versa. LBP-SDG in an optimal direction was compared with other features to prove that LBP-SDG is effective, and HSDG is a discriminative feature to recognize micro-expression. Furthermore, comparing with other methods, EVM+LBPSDG has state-of-the-art performance.

The relative recognition rate is mentioned in this paper for the most convenient description, where the relative recognition rate is the recognition rate of the LBP-SDG minus that of LBP-TOP. Thus, the effective directions are the directions in which the relative recognition rate is greater than 0 . Note that the radius parameters $\left(R_{X V}, R_{Y V}, R_{T V}, R_{X}\right.$, $R_{Y}$, and $R_{T}$ ) determine the time step and gradient value, so the study is based on these parameters.

\subsection{Setting}

All experiments reported the recognition rate (RR) under leave-one-subject-out (LOSO) crossvalidation. Experiments include the following three parts: 1) LBP-SDG in 18 directions was tested for the studied aim; 2) LBP-SDG was compared with other features under the same conditions; 3 ) LBP-SDG in the optimal direction was compared with other methods (including the state of the art method) by showing their recognition rate directly. All experiments were performed on SMIC and CASME II databases.

The SMIC database is the first spontaneous micro-expression database published by the University of Oulu, and the participants come from multiple countries. The SMIC database includes three subsets collected by three types of cameras: highspeed (HS), normal visual (VIS), and near-infrared (NIS). The SMIC-HS database is a subset of SMIC with the largest sample number and frame rate and was adopted in our experiment. The SMIC-HS database contains 164 samples and is divided into three categories: "Positive", "Negative" and "Surprise". Support Vector Machine with the linear kernel was employed as the classifier. The block strategy was adopted, and each video was divided into 8 $* 8 * 2$ blocks 51 . The frames number was unified to 10 using TIM and each frame was resized to 168 * 136 (grayscale image); thus, each video was normalized to $168 * 136 * 10$. The quantized number $\mathrm{N}$ was set to 2 , which is equivalent to binarization according to Equation 8. Also, $R_{V}=R_{X V}=R_{Y V}=$ $R_{T V}=1,2,3,4, R=R_{X}=R_{Y}=R_{T}=1,2,3,4$, and Alpha $=8,9,10,11,12$.

The CASME II database is the largest spontaneous micro-expression database published by the Institute of Psychology, Chinese Academy of Sciences, and the participants come from China. The CASME II database contains 255 samples (26 subjects) and is divided into seven categories. The two types of samples ( "Sadness" and "Fear" expressions) are relatively small compared to other types, so they are deleted. Eventually, five types of expression samples ("Happiness", "Disgust", "Repression", "Surprise", and "Others") are selected, and the selected sample size is 246. Support Vector Machine with the Chi-Square kernel was employed as the classifier. Each video was divided into $5 * 5$ * 1 blocks. The number of frames adopts the raw number without using TIM, and each frame was un-resized to unify size. The quantized number $\mathrm{N}$ was set to 2 . $R_{X V}=R_{Y V}=1, R_{V}=R_{T V}=$ $2,3, \ldots, 8, R_{X}=R_{Y}=1, R=R_{T}=2,3, \ldots, 8$, and Alpha $=17,20,23,26,29$. Also, the uniform LBP [52] was employed to calculate LBP-TOP.

As for the determination of alpha values, first, we tested the alpha values (from 1 to 30 ) for LBP-TOP, and found the optimal alpha value for LBP-TOP; second, we selected the representative alpha values around the optimal alpha value (including the optimal alpha value), and under these selected alpha values, the recognition rate of LBP-TOP first increases and then decreases; finally, different features were verified under these selected alpha values.

\subsection{The Study of Movement Feature in Different Directions}

Our experimental aim is to study movement feature in different directions. In order to analyze the studied aims in detail and comprehensively, this experiment was carried out under two settings: using EVM and not using EVM. 


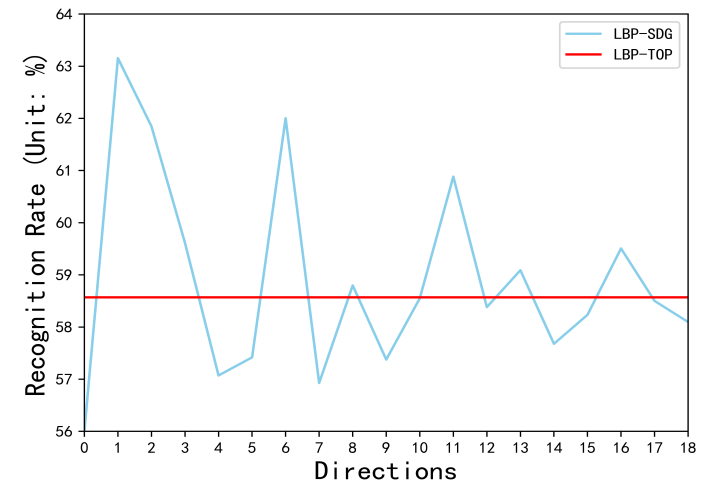

(a) The recognition rate under not using EVM.

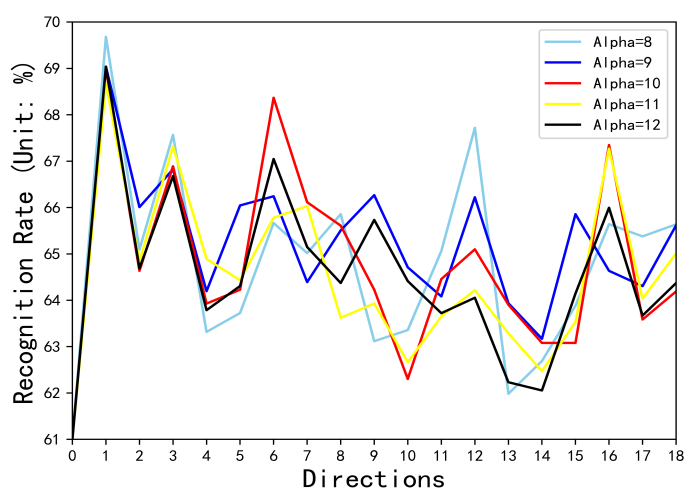

(c) The recognition rate under using EVM.

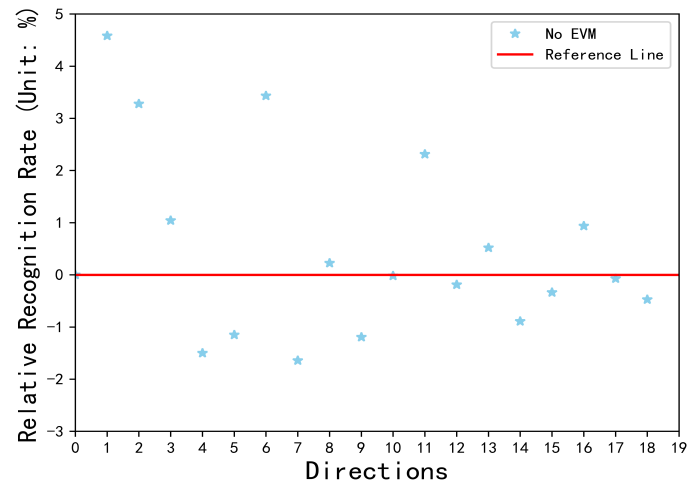

(b) The relative recognition rate under not using EVM.

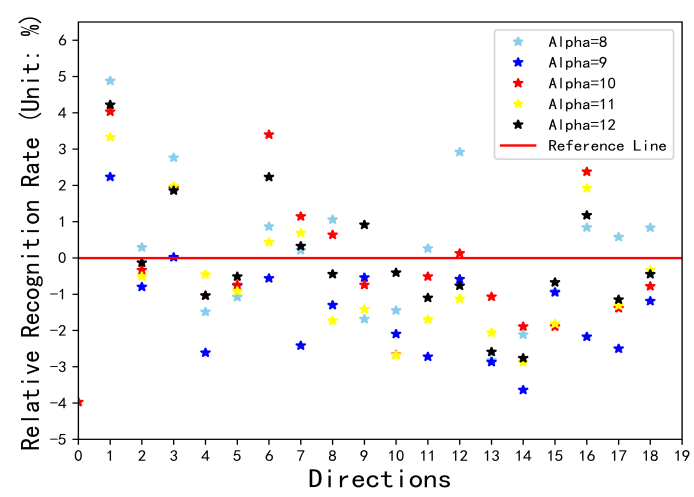

(d) The relative recognition rate under using EVM.

Figure 3: The SMIC-HS database: the line chart of the recognition rate and the dot charts of relative recognition rate in 18 directions.

The experiment results not using EVM are reported and analyzed in this paragraph. Table 5 (see appendices) shows the recognition rate of LBPSDG in 18 directions. The maximum recognition rate $(58.57 \%$ on $\mathrm{SMIC}, 51.48 \%$ on CASME II) of LBP-TOP was used as a reference line. In Figure $3(\mathrm{a})$ and $4(\mathrm{a})$, the 18 points (recognition rates in 18 directions) are connected to form a line chart; in Figure 3(b) and 4(b), the relative recognition rate is displayed as 18 independent points. As shown in the two figures, the movement feature (in directions (1), (2) and (6) on SMIC-HS, and (1) (2) (9) (11) (18) on CASME II) is very effective; the movement feature (in directions (3), (8), (11), (13) and (16) on SMIC-HS, and (3), (5), (6), (8), (13), (14) and (15) on CASME II) is suboptimal; and the movement feature in other directions not only cannot provide discriminative feature information but also produces disruptive feature information.

The experimental results using EVM are reported and analyzed in this paragraph. Table 6 and 7 (see appendices) show the recognition rates of LBP-SDG in 18 directions under different Alpha values. As shown in Figure 3(c) and 4(c), under different Alpha values, the changing trend with the change of direction is consistent in the line chart, and in Figure $3(\mathrm{c})$ the recognition rate in directions (1), (3), (6), (12) and (16) reaches the local peak, and in direction (2), (4), (14) and (17), it reaches the local trough, which means that the effective directions also tend to be consistent under different Alpha values. As EVM under different Alpha val- 


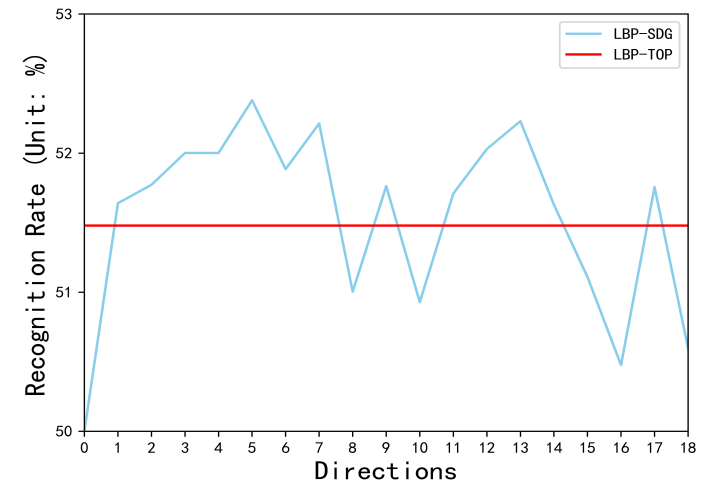

(a) The recognition rate under not using EVM.

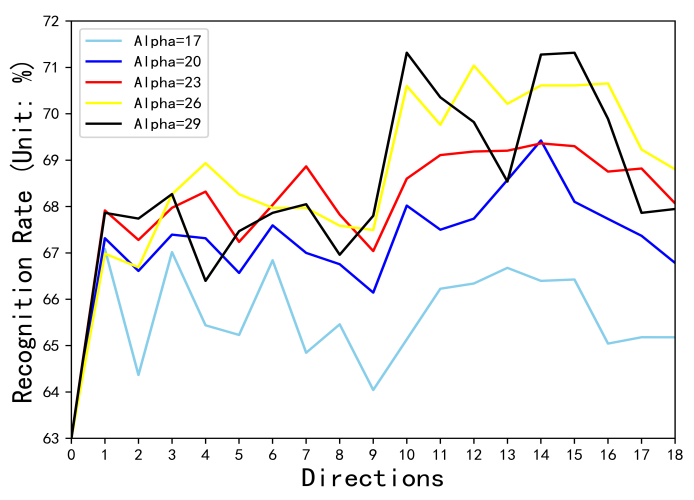

(c) The recognition rate under using EVM.

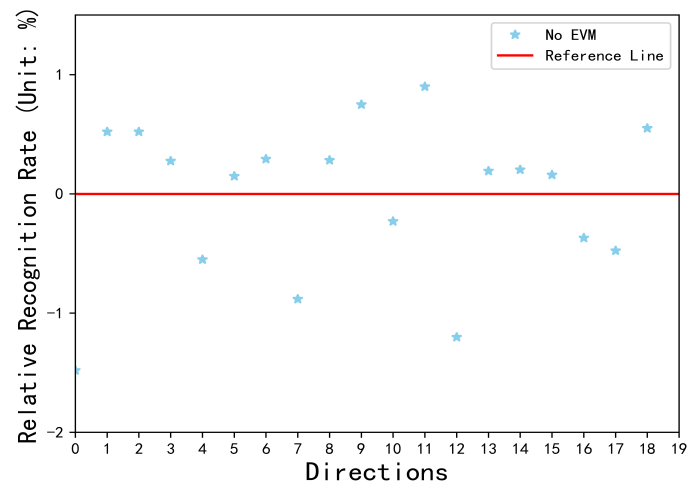

(b) The relative recognition rate under not using EVM.

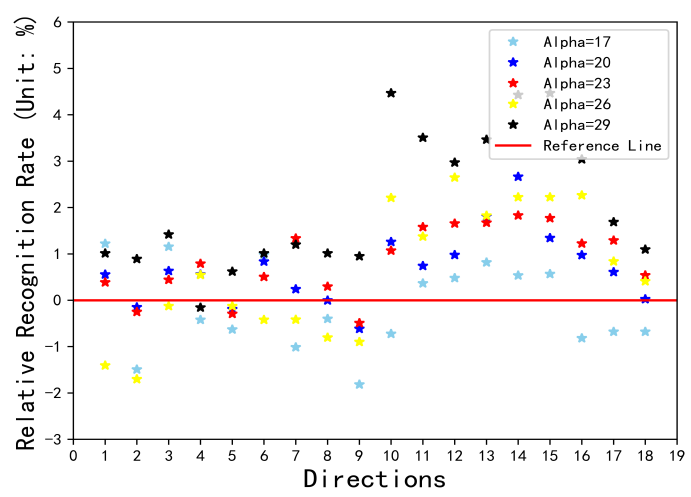

(d) The relative recognition rate under using EVM.

Figure 4: The CASME II database: the line chart of the recognition rate and the dot charts of relative recognition rate in 18 directions.

ues can affect the movement features, the effective directions under different Alpha values have somewhat instability, but the overall trends to be consistent. As shown in Figure 3(d) and 4(d), with the red line as a reference(the recognition rate of LBP-TOP as shown in Table 1), the movement features in direction (1) on SMIC-HS and (11) - (15) on CASME II are very effective, the one in directions (3), (6), (7) and (16) on SMIC-HS, and (1), (3), (6), (10), (16) and (17) on CASME II is suboptimal, and the one in other directions is invalid and even disruptive.

Overall, the studied results on different databases are different. The difference is mainly due to the differences between different databases, such as the country from which the participants came, the col- lection equipment and environment, etc. These differences and different parameters lead to different effective directions for the two databases and prompted the most suitable Alpha value to be different. Thus, on SMIC-HS, LBP-SDG in directions (1), (3), (6) and (16) outperforms LBPTOP whether EVM is employed or not, and the effective directions are (1), (3), (6) and (16). On the CASME II database, the studied results were divided into two parts: not using EVM, the movement feature in directions (1), (2), (9), (11) and (18) is very effective, and using EVM, the one in directions (11) - (15) is effective. Furthermore, the optimal direction is direction (1) on SMICHS and around (14) on CASME II, which means: on SMIC-HS, the variations of the pixel values 
along the time axis are optimal for distinguishing micro-expressions; on CASME II, the variations of the pixel values in the upward or horizontal direction are very effective for distinguishing the microexpression.

\subsection{Comparison with Other Features}

This experimental aim is to prove the effectiveness of LBP-SDG and to prove that HSDG can provide discriminative feature information. All experiments used EVM, and the results of LBP-SDG are in optimal directions. We compared LBP-SDG with other features, including LVP-TOP, GDLBPLVP-TOP, LBP-SIP, SIP-SDG, LBP-TOP. LVP and GDLBP-LVP are the LBP variants and were used effectively in face recognition. LVP-TOP and GDLBP-LVP-TOP can be obtained by extracting LVP 53] and GDLBP-LVP 54 on three orthogonal planes for micro-expression recognition, and SIPSDG can be obtained by concatenating LBP-SIP with HSDG. Table 1 shows the comparative results on both databases, and Table 2 shows the feature dimension of different features.

Overall, in comparison with other features, LBPSDG has the best performance under every Alpha value. In detail, SIP-SDG and LBP-SDG have better performance than LBP-SIP and LBP-TOP, respectively, which demonstrates that HSDG provides discriminative feature information. As the performance of SIP-SDG is similar to that of LBP-TOP, and the feature dimension of SIPSDG is lower than that of LBP-TOP, SIP-SDG can be taken as an alternative features of LBPTOP; GDLBP-LVP-TOP concatenate LVP-TOP with three LBP-TOP, but in comparison with LBPTOP, both LVP-TOP and GDLBP-LVP-TOP have worse performance, which demonstrates the following: 1) the effective features in other fields may not be effective for micro-expression recognition, and may even worsen the recognition rate; and 2) the proposed HSDG not only has a low feature dimension but also can enhance the performance of LBPTOP/LBP-SIP, which shows that HSDG is a discriminative feature to represent micro-expressions. Furthermore, we compare our method with HOGTOP and HIGO-TOP using EVM, and the results of the two features can be found in 41. As shown in Table 3. LBP-SDG is superior to HOG-TOP and HIGO-TOP. In terms of the highest recognition rate, LBP-SDG outperforms these features on both databases.
We further compare the confusion matrices of LVP-TOP, GDLBP-LVP-TOP, LBP-SIP, SIPSDG, LBP-TOP and LBP-SDG in Figure 5 and 6 (see appendices). On SMIC, LBP-SDG does not have the best performance at recognizing a certain class of expression, while it has the best average performance over three classes. On CASME II, SIP-SDG has the best performance at recognizing "Happiness"; GDLBP-LVP-TOP has best performance at recognizing "Surprise"; LVP-TOP, LBP-SIP and LBP-TOP has best performance for recognizing "Others"; for the other two microexpressions("Disgust", "Repression"), all feature have poor performance, while LBP-SDG has the best performance ( $57 \%$ for "Disgust" and $56 \%$ for "Repression"); In terms of the average performance, LBP-SDG is optimal. Furthermore, according to Table 2, HSDG has a very small feature dimension, which makes the feature dimension of LBP-SDG (SIP-SDG) very close to that of LBP-TOP (LBP-SIP); LVP-TOP and GDLBPLVP-TOP have a very high feature dimension, which seriously reduces the speed of recognition.

\subsection{Comparison with Other Methods}

This section proves that EVM+LBP-SDG is competitive. The recognition rate of different methods is shown in Table 4 it can be seen that EVM+LBP-SDG has the best performance (69.68\% on SMIC-HS and $71.32 \%$ on CASME II). CEF is the state-of-the-art method in the traditional methods. Although CEF employs an additional model to integrate some processing, EVM+LBP-SDG still has better performance. LEARNet, SSSN and TSCNN are the state-ofthe-art methods in deep learning-based methods. First, as shown in Table 4. LEARNet has the highest recognition rate, while it adopts $\mathrm{N}$-fold cross-validation and not the mainstream crossvalidation method (LOSO); second, comparing SSSN, EVM+LBP-SDG shows only a slight advantage on CASME II, but on SMIC-HS, EVM+LBPSDG has an obvious advantage; third, in terms of recognition rate, TSCNN has the best results, but similar to SSSN, it needs to detect apex frame before recognizing micro-expressions, and in terms of inputs, TSCNN need to calculate optical flow, while our method does not; finally, the deep learningbased methods train a feature extractor and the classifier for each subject validation, while the traditional methods (including our method) uses the same feature extractor for all subject validation. 
Table 1: Comparison LBP-SDG with other features.

(a) On the SMIC-HS

\begin{tabular}{ccccccc}
\hline Alpha & LVP-TOP & GD-LVP-TOP & LBP-SIP & SIP-SDG & LBP-TOP & LBP-SDG \\
\hline 8 & $52.88 \%$ & $61.15 \%$ & $60.59 \%$ & $65.71 \%$ (1) & $64.80 \%$ & $\mathbf{6 9 . 6 8 \%}(1)$ \\
9 & $53.65 \%$ & $59.60 \%$ & $59.69 \%$ & $64.01 \%$ (1) & $66.81 \%$ & $\mathbf{6 9 . 0 4 \%}$ (1) \\
10 & $51.85 \%$ & $59.58 \%$ & $59.74 \%$ & $64.79 \%$ (1) & $64.97 \%$ & $\mathbf{6 7 . 8 6 \%}$ (1) \\
11 & $55.15 \%$ & $60.36 \%$ & $60.01 \%$ & $67.17 \%$ (1) & $65.35 \%$ & $\mathbf{6 8 . 6 7 \% ( 1 )}$ \\
12 & $55.04 \%$ & $59.09 \%$ & $58.54 \%$ & $66.62 \%$ (1) & $64.82 \%$ & $\mathbf{6 9 . 0 4 \%}(1)$ \\
\hline
\end{tabular}

(b) On the CASME II

\begin{tabular}{ccccccc}
\hline Alpha & LVP-TOP & GD-LVP-TOP & LBP-SIP & SIP-SDG & LBP-TOP & \multicolumn{1}{c}{ LBP-SDG } \\
\hline 17 & $62.53 \%$ & $65.76 \%$ & $58.93 \%$ & $62.73 \%$ (15) & $65.86 \%$ & $\mathbf{6 7 . 0 8 \% ~ ( 1 ) ~}$ \\
20 & $62.27 \%$ & $66.78 \%$ & $61.55 \%$ & $64.08 \%$ (15) & $66.76 \%$ & $\mathbf{6 9 . 4 2 \%}$ (14) \\
23 & $65.48 \%$ & $66.41 \%$ & $62.92 \%$ & $64.28 \%$ (15) & $67.53 \%$ & $\mathbf{6 9 . 3 6 \%}(14)$ \\
26 & $64.90 \%$ & $66.20 \%$ & $63.50 \%$ & $65.32 \%$ (15) & $68.39 \%$ & $\mathbf{7 1 . 0 4 \%}(12)$ \\
29 & $65.24 \%$ & $66.37 \%$ & $64.06 \%$ & $67.61 \%$ (10) & $66.68 \%$ & $\mathbf{7 1 . 3 2 \%}$ (15) / (10) \\
\hline
\end{tabular}

Table 2: Comparison of feature dimension.

\begin{tabular}{ccc}
\hline Methods & CASME II & SMIC-HS \\
\hline HSDG & 50 & 256 \\
LVP-TOP & 17700 & 90624 \\
GD-LVP-TOP & 30975 & 109056 \\
LBP-SIP & 1500 & 7680 \\
SIP-SDG & 1550 & 7930 \\
LBP-TOP & 4425 & 6144 \\
LBP-SDG & 4475 & 6400 \\
\hline
\end{tabular}

Table 3: Comparison of accuracy rate between LBP-SDG, HOG-TOP and HIGO-TOP.

\begin{tabular}{ccc}
\hline Methods & CASME II & SMIC-HS \\
\hline HOG-TOP & 63.97 & 61.59 \\
HIGO-TOP & 67.21 & 68.29 \\
LBP-SDG & $\mathbf{7 1 . 3 2}$ & $\mathbf{6 9 . 6 8}$ \\
\hline
\end{tabular}

Table 4: Comparison of recognition rate between the proposed method and other methods.

\begin{tabular}{|c|c|c|}
\hline Method & SMIC-HS & CASME II \\
\hline FDM [36] & $54.88 \%$ & $45.93 \%$ \\
\hline STCLQP 28] & $64.02 \%$ & $58.39 \%$ \\
\hline STLBP-IP [29] & $57.93 \%$ & $59.51 \%$ \\
\hline TIM+STRBP 55 & $60.98 \%$ & $64.37 \%$ \\
\hline $\mathrm{CNN}+\mathrm{LSTM}[20]$ & - & $60.98 \%$ \\
\hline ELRCN-TE [56] & - & $52.44 \%$ \\
\hline STLBP-IP+KGSL 22 & $60.78 \%$ & $63.83 \%$ \\
\hline 3DFCNN 44 & $55.49 \%$ & $59.11 \%$ \\
\hline TIM+EVM+HIGO 41. & $68.29 \%$ & $67.21 \%$ \\
\hline LEARNet* 46 & - & $76.59 \%$ \\
\hline $\mathrm{SSSN}^{\curlyvee}$ [7] & 63.41 & $71.19 \%$ \\
\hline TSCNN $^{\curlyvee} 48$ & 72.74 & $74.05 \%$ \\
\hline CEF [18] & $68.90 \%$ & $70.85 \%$ \\
\hline The proposed method & $69.68 \%$ & $71.32 \%$ \\
\hline \multicolumn{3}{|c|}{$\begin{array}{l}\text { no result in the original paper } \\
\checkmark \text { the method uses the apex frame } \\
* \text { the results adopted different cross-validation. }\end{array}$} \\
\hline
\end{tabular}




\section{Future Works}

In this work, HSDGs in 18 directions were tested in turn to artificially select effective HSDGs, which spent a lot of time and didn't consider the multiple HSDGs simultaneously. In addition, different parameters and databases will lead to different conclusions about the effective HSDGs. In future works, we will solve it by employing the feature selection algorithm that can automatically select effective HSDGs and process 18 HSDGs simultaneously. According to the work in this paper, LBP-TOP and HSDG have different importance. Concretely, LBP-TOP is the main feature that provides abundant feature information, and HSDGs is the supplementary feature that provides the limited feature information and different HSDGs have different performance. Thus, this algorithm selects effective HSDGs under concatenating with LBPTOP and should consider the different importance of LBP-TOP and HSDG. In this way, we needn't test each HSDG in turn and can consider multiple HSDGs simultaneously. In addition, more discriminative features can be selected to recognize microexpressions, and the performance can further be improved.

\section{Conclusions}

This paper studies movement feature in different directions and proposes a new and low-dimensional single-direction movement feature HSDG. HSDG actively extracts the movement feature in a certain direction. First, the gradient values are calculated in a certain direction, then these values are quantified before performing histogram operations. Concatenating HSDG with LBP-TOP, we obtain LBP-SDG. In experiments, LBP-SDG in 18 directions was tested to ensure effective and optimal directions, and LBP-SDG in an optimal direction was compared with other features and methods. On SMIC-HS, the movement features along the time axis are optimal for distinguishing microexpressions; on CASME II, extracting the movement features in the upward or horizontal directions is very effective for distinguishing the microexpression. Comparing it with other features, LBPSDG in an optimal direction has the best performance. By comparing the changes of LBP-TOP and LBP-SIP before and after adding HSDG, it is proven that HSDG can provide discriminative feature information. Additionally, the results show that LBP-SDG+EVM outperforms state-of-the-art methods.

\section{Acknowledgements}

This work was partly supported by the Postgraduate Research and Practice Innovation Program of Jiangsu Province (Grant KYCX18_0899), partly by the National Natural Science Foundation of China (NSFC) under Grants 72074038, partly by the Key Research and Development Program of Jiangsu Province(Grant BE2016775), partly by the National Natural Science Foundation of China (NSFC) (Grants 61971236), partly by China Postdoctoral Science Foundation (Grant 2018M632348).

\section{References}

[1] E. Haggard, K. Isaacs, Methods of Research in Psychotherapy, 1966, pp. 154-165. doi:10.1007/ 978-1-4684-6045-2_14.

[2] P. Ekman, E. Rosenberg, Editors, What the face reveals: Basic and applied studies of spontaneous expression using the facial action coding system (facs) doi: 10.1093/acprof : oso/9780195179644.001.0001

[3] S. Porter, L. Brinke, Reading between the lies, Psychological science 19 (2008) 508-14. doi:10.1111/j. 1467-9280.2008.02116.x

[4] D. Matsumoto, H. Hwang, Evidence for training the ability to read microexpressions of emotion, Motivation and Emotion 35 (2011) 181-191. doi:10.1007/ s11031-011-9212-2

[5] W.-J. Yan, Q. Wu, Y.-H. Chen, J. Liang, X. Fu, How fast are the leaked facial expressions: The duration of micro-expressions, Journal of Nonverbal Behavior 37. doi:10.1007/s10919-013-0159-8

[6] P. Ekman, Lie Catching and Microexpressions, 2009, pp. 118-136. doi:10.1093/acprof: oso/9780195327939.003.0008.

[7] M. O'Sullivan, M. Frank, C. Hurley, J. Tiwana, Police lie detection accuracy: The effect of lie scenario, Law and Human Behavior 33 (2009) 530-538. doi:10.1007/ s10979-008-9166-4

[8] S. Weinberger, Airport security: Intent to deceive?, Nature 465 (2010) 412-5. doi:10.1038/465412a

[9] M. Frank, M. Herbasz, K. Sinuk, A. M. Keller, A. Kurylo, C. Nolan, I see how you feel: Training laypeople and professionals to recognize fleeting emotions, in: International Communication Association, 2009 .

[10] P. Ekman, Telling lies: clues to deceit in the marketplace, politics, and marriage.

[11] P. Ekman, Microexpression training tool (mett), University of California, San Francisco, CA. (2002).

[12] C. J. G. V. Frank, Mark G. Maccario, Behavior and security, Protecting Airline Passengers in the Age of Terrorism (2009) 86-106.

[13] W. Yan, X. Li, S. Wang, G. Zhao, Y. Liu, Y. Chen, $\mathrm{X}$. Fu, Casme ii: An improved spontaneous microexpression database and the baseline evaluation, PLOS 
ONE 9 (1) (2014) 1-8. doi:10.1371/journal.pone. 0086041

[14] X. Li, T. Pfister, X. Huang, G. Zhao, M. Pietikäinen, A spontaneous micro-expression database: Inducement, collection and baseline, in: 2013 10th IEEE International Conference and Workshops on Automatic Face and Gesture Recognition, FG 2013, 2013, pp. 1-6. doi:10.1109/FG.2013.6553717

[15] A. Davison, C. Lansley, N. Costen, K. Tan, M. H. Yap, Samm: A spontaneous micro-facial movement dataset, IEEE Transactions on Affective Computing 9 (1) (2018) 116-129. doi:10.1109/TAFFC. 2016.2573832

[16] H.-Y. Wu, M. Rubinstein, E. Shih, J. Guttag, F. Durand, W. Freeman, Eulerian video magnification for revealing subtle changes in the world, ACM Transactions on Graphics 31.

[17] Z. Zhou, G. Zhao, M. Pietikäinen, Towards a practical lipreading system, in: Proceedings of the IEEE Computer Society Conference on Computer Vision and Pattern Recognition, 2011, pp. 137-144. doi:10.1109/ CVPR.2011.5995345

[18] W. Peng, X. Hong, Y. Xu, G. Zhao, A boost in revealing subtle facial expressions: A consolidated eulerian framework, in: 14th IEEE International Conference on Automatic Face and Gesture Recognition (FG 2019), 2019. doi:10.1109/FG.2019.8756541

[19] L. Ngo, A.C., Phan, R.CW., S. J, Spontaneous subtle expression recognition: Imbalanced databases and solutions, in: Asian Conference on Computer Vision, 2015, pp. 33-48. doi:10.1007/978-3-319-16817-3_3

[20] D. Kim, W. Baddar, Y. Ro, Micro-expression recognition with expression-state constrained spatio-temporal feature representations, in: Acm on Multimedia Conference, 2016, pp. 382-386. doi:10.1145/2964284. 2967247

[21] X. Jia, X. Ben, H. Yuan, K. Kpalma, W. Meng, Macroto-micro transformation model for micro-expression recognition, Journal of Computational Science (2017) 289-297doi:10.1016/j.jocs.2017.03.016.

[22] Y. Zong, X. Huang, W. Zheng, Z. Cui, G. Zhao, Learning from hierarchical spatiotemporal descriptors for micro-expression recognition, IEEE Transactions on Multimedia 20 (2018) 3160-3172. doi:10.1109/TMM. 2018.2820321

[23] S. Zhang, B. Feng, Z. Chen, X. Huang, MicroExpression Recognition by Aggregating Local SpatioTemporal Patterns, Springer International Publishing, 2017. doi:10.1007/978-3-319-51811-4_52

[24] T. Ojala, M. Pietikäinen, D. Harwood, A comparative study of texture measures with classification based on feature distributions, Pattern Recognition 29 (1996) 5159. doi:10.1016/0031-3203(95)00067-4

[25] G. Zhao, M. Pietikäinen, Dynamic texture recognition using local binary patterns with an application to facial expressions, IEEE Transactions on Pattern Analysis and Machine Intelligence 29 (6) (2007) 915-928. doi:10.1109/TPAMI.2007.1110

[26] Y. Wang, J. See, R. Phan, Y.-H. Oh, Lbp with six intersection points: Reducing redundant information in lbptop for micro-expression recognition, in: ACCV, Vol 9003, 2015. doi:10.1007/978-3-319-16865-4_34.

[27] Y. Wang, J. See, R. Phan, Y.-H. Oh, Efficient spatiotemporal local binary patterns for spontaneous facial micro-expression recognition, PloS one 10 (2015) e0124674. doi:10.1371/journal.pone.0124674
[28] X. Huang, G. Zhao, X. Hong, W. Zheng, M. Pietikäinen, Spontaneous facial micro-expression analysis using spatiotemporal completed local quantized patterns, Neurocomputing 175 (2015) 564-578. doi:10.1016/j.neucom.2015.10.096

[29] X. Huang, S.-J. Wang, G. Zhao, M. Pietikäinen, Facial micro-expression recognition using spatiotemporal local binary pattern with integral projection, in: 2015 IEEE International Conference on Computer Vision Workshop, 2015, pp. 1-9. doi:10.1109/ICCVW.2015.10

[30] X. Huang, S. Wang, X. Liu, G. Zhao, X. Feng, M. Pietikäinen, Discriminative spatiotemporal local binary pattern with revisited integral projection for spontaneous facial micro-expression recognition, IEEE Transactions on Affective Computing 10 (1) (2019) 3247. doi:10.1109/TAFFC. 2017.2713359

[31] Y. Wang, J. See, Y.-H. Oh, R. C.-W. Phan, R. Rahulamathavan, H.-C. Ling, S.-W. Tan, X. Li, Effective recognition of facial micro-expressions with video motion magnification, Multimedia Tools and Applications 76 (2017) 21665-21690. doi:10.1007/ s11042-016-4079-6

[32] B. Horn, B. Schunck, Determining optical flow, Artificial Intelligence 17 (1981) 185-203. doi:10.1016/ 0004-3702 (81) 90024-2

[33] S.-T. Liong, R. Phan, J. See, Y.-H. Oh, K. Wong, Optical strain based recognition of subtle emotions, in: 2014 International Symposium on Intelligent Signal Processing and Communication Systems (ISPACS), 2015, pp. 180-184. doi:10.1109/ISPACS.2014.7024448

[34] S. Liong, J. See, R. Phan, A. C. Le Ngo, Y.-H. Oh, K. Wong, Subtle expression recognition using optical strain weighted features, in: Computer VisionACCV 2014 Workshops, Revised Selected Papers, Part II, Vol. 9009, 2014, pp. 644-657. doi:10.1007/ 978-3-319-16631-5_47

[35] S. Liong, J. See, R. Phan, K. Wong, Less is more: Micro-expression recognition from video using apex frame, Signal Processing: Image Communication 62 (2016) 82-92. doi:10.1016/j.image.2017.11.006

[36] F. Xu, J. Zhang, J. Wang, Microexpression identification and categorization using a facial dynamics map, IEEE Transactions on Affective Computing 8 (2017) 254-267. doi:10.1109/TAFFC.2016.2518162

[37] Y.-J. Liu, J.-K. Zhang, W.-J. Yan, S.-J. Wang, G. Zhao, $\mathrm{X}$. Fu, A main directional mean optical flow feature for spontaneous micro-expression recognition, IEEE Transactions on Affective Computing 7 (4) (2016) 299-310. doi:10.1109/TAFFC.2015.2485205

[38] Y.-J. Liu, B.-J. Li, Y.-K. Lai, Sparse mdmo: Learning a discriminative feature for spontaneous micro-expression recognition, IEEE Transactions on Affective Computing PP (2018) 1-1. doi:10.1109/TAFFC. 2018.2854166

[39] S. L. Happy, A. Routray, Fuzzy histogram of optical flow orientations for micro-expression recognition, IEEE Transactions on Affective Computing 10 (3) (2019) 394-406. doi:10.1109/TAFFC.2017.2723386

[40] S. Polikovsky, Y. Kameda, Y. Ohta, Facial microexpressions recognition using high speed camera and 3d-gradient descriptor, in: 3rd International Conference on Imaging for Crime Detection and Prevention (ICDP 2009), 2010, pp. 1 - 6. doi:10.1049/ic.2009.0244

[41] X. Li, X. Hong, A. Moilanen, X. Huang, T. Pfister, G. Zhao, M. Pietikäinen, Towards reading hidden emotions: A comparative study of spontaneous micro- 
expression spotting and recognition methods, IEEE Transactions on Affective Computing 9 (4) (2018) 563577. doi:10.1109/TAFFC.2017.2667642

[42] Y. Li, X. Huang, G. Zhao, Can micro-expression be recognized based on single apex frame?, in: IEEE 2018 25th IEEE International Conference on Image Processing (ICIP), 2018, pp. 3094-3098. doi:10.1109/ICIP. 2018.8451376

[43] S.-J. Wang, B.-J. Li, Y.-J. Liu, W.-J. Yan, X. Ou, X. Huang, F. Xu, X. Fu, Micro-expression recognition with small sample size by transferring long-term convolutional neural network, Neurocomputing 312 (2018) 251-262. doi:10.1016/j.neucom.2018.05.107

[44] S. J. L. W. Li Jing, Wang Yandan, Micro-expression recognition based on $3 \mathrm{~d}$ flow convolutional neural network, Pattern Analysis and Applications 22 (2018) 1331-1339. doi:10.1007/s10044-018-0757-5.

[45] S. Liong, Y. Gan, J. See, H.-Q. Khor, Y.-C. Huang, Shallow triple stream three-dimensional cnn (ststnet) for micro-expression recognition, in: 2019 14th IEEE International Conference on Automatic Face and Gesture Recognition (FG 2019), 2019, pp. 1-5. doi:10. 1109/FG.2019.8756567.

[46] M. Verma, S. Vipparthi, G. Singh, S. Murala, Learnet dynamic imaging network for micro expression recognition, IEEE Transactions on Image Processing (2019) Fdoi:10.1109/TIP. 2019.2912358

[47] H.-Q. Khor, J. See, S. Liong, R. Phan, W. Lin, Dualstream shallow networks for facial micro-expression recognition, in: 2019 IEEE International Conference on Image Processing (ICIP), 2019, pp. 36-40. doi: 10.1109/ICIP.2019.8802965

[48] B. Song, K. Li, Y. Zong, Z. Jie, W. Zheng, J. Shi, L. Zhao, Recognizing spontaneous micro-expression using a three-stream convolutional neural network, IEEE Access PP (2019) 1-1. doi:10.1109/ACCESS. 2019. 2960629

[49] K. Goh, C. Ng, L. Lim, U. Sheikh, Micro-expression recognition: an updated review of current trends, challenges and solutions, The Visual Computer 36 (2018) 1-1. doi:10.1007/s00371-018-1607-6

[50] N. Dalal, B. Triggs, Histograms of oriented gradients for human detection, in: IEEE Conference on Computer Vision and Pattern Recognition (CVPR 2005), Vol. 1, 2005, pp. 886-893. doi:10.1109/CVPR.2005.177

[51] X. Hong, Y. Xu, G. Zhao, Lbp-top: a tensor unfolding revisit, in: ACCV Workshop on "Spontaneous Facial Behavior Analysis", 2016. doi:10.1007/ 978-3-319-54407-6_34

[52] T. Ojala, M. Pietikainen, T. Maenpaa, Multiresolution gray-scale and rotation invariant texture classification with local binary patterns, IEEE Transactions on Pattern Analysis and Machine Intelligence 24 (7) (2002) 971-987. doi:10.1109/tpami.2002.1017623.

[53] K. Fan, T. Hung, A novel local pattern descriptor-local vector pattern in high-order derivative space for face recognition, IEEE Transactions on Image Processing 23 (7) (2014) 2877-2891. doi:10.1109/TIP. 2014.2321495

[54] S. Chakraborty, S. K. Singh, P. Chakraborty, Performance enhancement of local vector pattern with generalized distance local binary pattern for face recognition, in: 2015 IEEE UP Section Conference on Electrical Computer and Electronics, 2015, pp. 1-5. doi: 10.1109/UPCON. 2015.7456681.
[55] X. Huang, G. Zhao, Spontaneous facial microexpression analysis using spatiotemporal local radonbased binary pattern, in: International Conference on The Frontiers and Advances in Data Science, 2016, pp. 382-386. doi:10.1109/FADS.2017.8253219

[56] H.-Q. Khor, J. See, R. Phan, W. Lin, Enriched longterm recurrent convolutional network for facial microexpression recognition, in: 2018 13th IEEE International Conference on Automatic Face and Gesture Recognition, 2018, pp. 667-674. doi:10.1109/FG.2018. 00105

\section{Appendices}


Table 5: The recognition rate of LBP-SDG in 18 directions under not using EVM.

(a) On the SMIC-HS

\begin{tabular}{cccc}
\hline DT & $\mathrm{RR}\left(R_{V}, R\right)$ & $\mathrm{DT}$ & $\mathrm{RR}\left(R_{V}, R\right)$ \\
\hline (1) & $\mathbf{6 3 . 1 5 \%}(1,2)$ & (10) & $58.55 \%(1,4)$ \\
(2) & $61.85 \%(3,3)$ & (11) & $60.88 \%(3,3)$ \\
(3) & $59.61 \%(1,1)$ & (12) & $58.38 \%(1,1)$ \\
(4) & $57.07 \%(1,1)$ & (13) & $59.09 \%(3,1)$ \\
(5) & $57.42 \%(2,1)$ & (14) & $57.68 \%(1,1)$ \\
(6) & $62.00 \%(1,1)$ & (15) & $58.23 \%(1,1)$ \\
(7) & $56.93 \%(1,1)$ & (16) & $59.51 \%(1,2)$ \\
(8) & $58.80 \%(2,1)$ & (17) & $58.50 \%(2,4)$ \\
(9) & $57.38 \%(1,2)$ & (18) & $58.10 \%(1,2)$ \\
\hline
\end{tabular}

(b) On the CASME II

\begin{tabular}{cccc}
\hline DT & $\mathrm{RR}\left(R_{V}, R\right)$ & $\mathrm{DT}$ & $\mathrm{RR}\left(R_{V}, R\right)$ \\
\hline (1) & $52.00 \%(5,3-4)$ & (10) & $51.88 \%(5,4)$ \\
(2) & $52.00 \%(5,2-35)$ & (11) & $\mathbf{5 2 . 3 8 \% ( 8 , 5 )}$ \\
(3) & $51.79 \%(4,8)$ & (12) & $50.48 \%(4,8)$ \\
(4) & $50.93 \%(3,2-3)$ & (13) & $52.21 \%(4,7)$ \\
(5) & $51.63 \%(5,7)$ & (14) & $51.71 \%(5,7)$ \\
(6) & $51.77 \%(5,3)$ & (15) & $51.65 \%(5,8)$ \\
(7) & $50.60 \%(4,6-8)$ & (16) & $51.11 \%(7,3-7)$ \\
(8) & $51.76 \%(4,8)$ & (17) & $51.00 \%(4,2-3)$ \\
(9) & $52.23 \%(5,2)$ & (18) & $52.03 \%(5,4-6)$ \\
\hline
\end{tabular}


Table 6: The SMIC-HS database: the recognition rate of LBP-SDG in 18 directions under different Alpha values.

(a) Alpha $=8$

\begin{tabular}{cccc}
\hline DT & $\mathrm{RR}\left(R_{V}, R\right)$ & $\mathrm{DT}$ & $\mathrm{RR}\left(R_{V}, R\right)$ \\
\hline (1) & $\mathbf{6 9 . 6 8 \%}(3,3)$ & 10 & $63.36 \%(3,3)$ \\
(2) & $65.10 \%(3,3)$ & 11 & $65.05 \%(3,4)$ \\
(3) & $67.57 \%(3,2)$ & 12 & $67.72 \%(3,1)$ \\
(4) & $63.32 \%(3,1)$ & 13 & $61.98 \%(3,1)$ \\
(5) & $63.72 \%(3,1)$ & 14 & $62.69 \%(3,1)$ \\
6 & $65.67 \%(3,2)$ & 15 & $63.88 \%(3,2)$ \\
(7) & $65.02 \%(3,1)$ & 16 & $65.64 \%(3,2)$ \\
8 & $65.86 \%(3,2)$ & 17 & $65.38 \%(3,1)$ \\
(9) & $63.12 \%(3,2)$ & 18 & $65.64 \%(3,1)$ \\
\hline
\end{tabular}

(c) Alpha $=10$

\begin{tabular}{cccc}
\hline DT & $\mathrm{RR}\left(R_{V}, R\right)$ & $\mathrm{DT}$ & $\mathrm{RR}\left(R_{V}, R\right)$ \\
\hline 1 & $\mathbf{6 9 . 0 0 \% ( 3 , 3 )}$ & 10 & $62.30 \%(3,4)$ \\
(2) & $64.64 \%(3,3)$ & 11 & $64.46 \%(3,4)$ \\
(3) & $66.89 \%(3,2)$ & 12 & $65.10 \%(3,1)$ \\
(4) & $63.93 \%(4,1)$ & 13 & $63.90 \%(3,3)$ \\
(5) & $64.23 \%(3,1)$ & 14 & $63.08 \%(3,1)$ \\
(6) & $68.37 \%(3,3)$ & 15 & $63.08 \%(3,1)$ \\
(7) & $66.11 \%(3,1)$ & 16 & $67.35 \%(3,3)$ \\
8 & $65.61 \%(3,2)$ & 17 & $63.58 \%(4,1)$ \\
(9) & $64.23 \%(3,2)$ & 18 & $64.19 \%(3,1)$ \\
\hline
\end{tabular}

(b) Alpha $=9$

\begin{tabular}{cccc}
\hline DT & $\mathrm{RR}\left(R_{V}, R\right)$ & $\mathrm{DT}$ & $\mathrm{RR}\left(R_{V}, R\right)$ \\
\hline (1) & $\mathbf{6 9 . 0 4 \%}(3,3)$ & 10 & $64.71 \%(3,2)$ \\
(2) & $66.01 \%(3,1)$ & 11 & $64.08 \%(3,2)$ \\
(3) & $66.83 \%(3,3)$ & 12 & $66.22 \%(3,1)$ \\
(4) & $64.19 \%(3,1)$ & 13 & $63.94 \%(3,4)$ \\
(5) & $66.05 \%(3,1)$ & 14 & $63.17 \%(3,2)$ \\
(6) & $66.24 \%(3,2)$ & 15 & $65.86 \%(3,1)$ \\
$(7$ & $64.39 \%(3,1)$ & 16 & $64.63 \%(3,2)$ \\
8 & $65.51 \%(3,2)$ & 117 & $64.30 \%(3,2)$ \\
(9) & $66.27 \%(3,2)$ & 18 & $65.12 \%(3,1)$ \\
\hline
\end{tabular}

(d) Alpha $=11$

\begin{tabular}{cccc}
\hline DT & $\mathrm{RR}\left(R_{V}, R\right)$ & $\mathrm{DT}$ & $\mathrm{RR}\left(R_{V}, R\right)$ \\
\hline 1 & $\mathbf{6 8 . 6 7 \%}(3,3)$ & 10 & $62.66 \%(3,4)$ \\
(2) & $64.84 \%(3,2)$ & 11 & $63.65 \%(3,2)$ \\
(3) & $67.32 \%(3,1)$ & 12 & $64.22 \%(3,2)$ \\
(4) & $64.89 \%(4,1)$ & 13 & $63.29 \%(3,3)$ \\
(5) & $64.43 \%(3,1)$ & 114 & $62.47 \%(4,1)$ \\
(6) & $65.78 \%(3,2)$ & 15 & $63.53 \%(3,1)$ \\
(7) & $66.03 \%(3,1)$ & 16 & $67.27 \%(3,2)$ \\
8 & $63.62 \%(3,3)$ & 17 & $64.04 \%(3,3)$ \\
(9) & $63.93 \%(3,2)$ & 18 & $65.01 \%(3,1)$ \\
\hline
\end{tabular}

(e) Alpha=12

\begin{tabular}{cccc}
\hline DT & $\mathrm{RR}\left(R_{V}, R\right)$ & $\mathrm{DT}$ & $\mathrm{RR}\left(R_{V}, R\right)$ \\
\hline (1) & $\mathbf{6 9 . 0 4 \%}(3,3)$ & 10 & $63.41 \%$ \\
(2) & $64.69 \%(4,4)$ & 11 & $63.72 \%(4,2)$ \\
(3) & $66.68 \%(3,2)$ & 12 & $64.05 \%(3,1)$ \\
(4) & $63.79 \%(4,1)$ & 13 & $62.23 \%(3,3)$ \\
(5) & $64.31 \%(3,2)$ & 14 & $62.06 \%(4,1)$ \\
(6) & $67.05 \%(3,3)$ & 15 & $64.15 \%(4,1)$ \\
(7) & $65.15 \%(4,1)$ & 16 & $65.99 \%(3,2)$ \\
8 & $64.37 \%(3,2)$ & 17 & $63.67 \%(3,4)$ \\
(9) & $65.73 \%(3,3)$ & 18 & $64.37 \%(3,1)$ \\
\hline
\end{tabular}


Table 7: The CASME II database: the recognition rate of LBP-SDG in 18 directions under different Alpha values.

(a) Alpha =17

\begin{tabular}{ccrc}
\hline DT & $\mathrm{RR}\left(R_{V}, R\right)$ & $\mathrm{DT}$ & $\mathrm{RR}\left(R_{V}, R\right)$ \\
\hline (1) & $\mathbf{6 7 . 0 8 \% ( 4 , 8 )}$ & 10 & $65.14 \%(5,4)$ \\
(2) & $64.37 \%(4,24)$ & 111 & $66.23 \%(4,4)$ \\
(3) & $67.02 \%(6,8)$ & 12 & $66.34 \%(4,8)$ \\
(4) & $65.44 \%(5,2)$ & 13 & $66.68 \%(4,8)$ \\
(5) & $65.23 \%(6,8)$ & 114 & $66.40 \%(4,8)$ \\
(6) & $66.84 \%(6,8)$ & 15 & $66.43 \%(6,8)$ \\
(7) & $64.85 \%(5,3)$ & 116 & $65.04 \%(5,4)$ \\
(8) & $65.46 \%(6,7)$ & 117 & $65.18 \%(4,8)$ \\
(9) & $64.04 \%(4,5)$ & 18 & $65.18 \%(4,3)$ \\
\hline
\end{tabular}

(c) Alpha $=23$

\begin{tabular}{cccc}
\hline DT & $\mathrm{RR}\left(R_{V}, R\right)$ & $\mathrm{DT}$ & $\mathrm{RR}\left(R_{V}, R\right)$ \\
\hline (1) & $67.92 \%(5,2)$ & 10 & $68.60 \%(4,6)$ \\
(2) & $67.28 \%(8,4)$ & 11 & $69.11 \%(7,4)$ \\
(3) & $67.97 \%(8,6)$ & 12 & $69.19 \%(4,2)$ \\
(4) & $68.32 \%(4,2)$ & 13 & $69.20 \%(4,6)$ \\
(5) & $67.24 \%(7,6)$ & 114 & $\mathbf{6 9 . 3 6 \%}(5,8)$ \\
(6) & $68.03 \%(7,3)$ & 15 & $69.30 \%(3,8)$ \\
(7) & $68.87 \%(4,2)$ & 16 & $68.75 \%(5,5)$ \\
(8) & $67.83 \%(5,2)$ & 17 & $68.82 \%(6,7)$ \\
(9) & $67.04 \%(6,3)$ & 18 & $68.07 \%(7,5)$ \\
\hline
\end{tabular}

(b) Alpha $=20$

\begin{tabular}{cccc}
\hline DT & $\mathrm{RR}\left(R_{V}, R\right)$ & $\mathrm{DT}$ & $\mathrm{RR}\left(R_{V}, R\right)$ \\
\hline (1) & $67.32 \%(6,7)$ & 10 & $68.02 \%(6,5)$ \\
(2) & $66.61 \%(6,3)$ & 111 & $67.50 \%(5,5)$ \\
(3) & $67.39 \%(5,7)$ & 112 & $67.74 \%(4,4)$ \\
(4) & $67.32 \%(6,8)$ & 13 & $68.57 \%(6,6)$ \\
(5) & $66.57 \%(6,8)$ & 114 & $\mathbf{6 9 . 4 2 \%}(4,8)$ \\
(6) & $67.59 \%(5,8)$ & 15 & $68.10 \%(4,8)$ \\
(7) & $67.00 \%(6,8)$ & 16 & $67.73 \%(4,6)$ \\
(8) & $66.76 \%(4,4)$ & 117 & $67.37 \%(4,4)$ \\
(9) & $66.14 \%(4,3)$ & (18) & $66.78 \%(8,4)$ \\
\hline
\end{tabular}

(d) Alpha $=26$

\begin{tabular}{cccc}
\hline DT & $\mathrm{RR}\left(R_{V}, R\right)$ & $\mathrm{DT}$ & $\mathrm{RR}\left(R_{V}, R\right)$ \\
\hline (1) & $66.98 \%(5,2)$ & 10 & $70.60 \%(6,8)$ \\
(2) & $66.69 \%(5,3)$ & 11 & $69.76 \%(5,4)$ \\
(3) & $68.27 \%(5,3)$ & 12 & $\mathbf{7 1 . 0 4 \% ( 5 , 8 )}$ \\
(4) & $68.93 \%(5,2)$ & 13 & $70.22 \%(6,5)$ \\
(5) & $68.27 \%(5,4)$ & 14 & $70.61 \%(5,8)$ \\
(6) & $67.97 \%(5,2)$ & 15 & $70.61 \%(5,8)$ \\
(7) & $67.97 \%(5,3)$ & 16 & $70.65 \%(6,6)$ \\
(8) & $67.59 \%(5,2)$ & 117 & $69.23 \%(5,4)$ \\
(9) & $67.49 \%(7,2)$ & 18 & $68.80 \%(5,5)$ \\
\hline
\end{tabular}

(e) Alpha $=29$

\begin{tabular}{cccc}
\hline DT & $\mathrm{RR}\left(R_{V}, R\right)$ & $\mathrm{DT}$ & $\mathrm{RR}\left(R_{V}, R\right)$ \\
\hline (1) & $67.86 \%(7,4)$ & 10 & $\mathbf{7 1 . 3 2 \%}(5,8)$ \\
(2) & $67.74 \%(7,2)$ & 111 & $70.36 \%(5,8)$ \\
(3) & $68.27 \%(7,8)$ & 12 & $69.82 \%(6,8)$ \\
(4) & $66.69 \%(7,4)$ & 13 & $70.32 \%(5,8)$ \\
(5) & $67.47 \%(8,4)$ & 14 & $71.28 \%(5,8)$ \\
(6) & $67.86 \%(7,4)$ & 15 & $71.32 \%(5,8)$ \\
(7) & $68.05 \%(5,4)$ & 116 & $69.89 \%(5,6)$ \\
8 & $67.86 \%(7,2)$ & 117 & $68.54 \%(5,8)$ \\
(9) & $67.80 \%(5,3)$ & 18 & $67.95 \%(5,8)$ \\
\hline
\end{tabular}




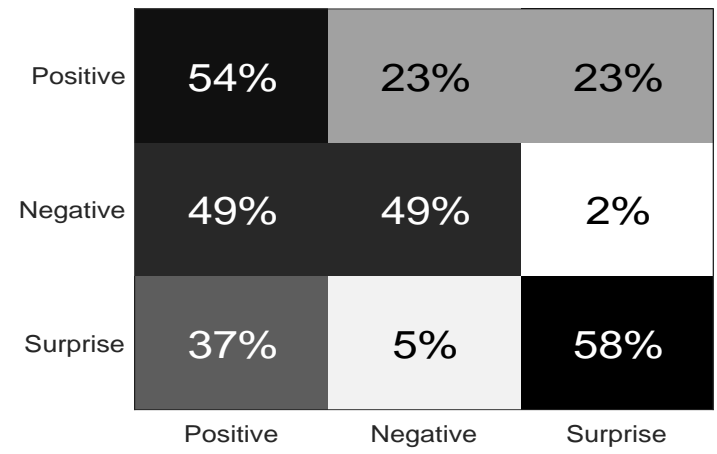

(a) LVP-TOP.

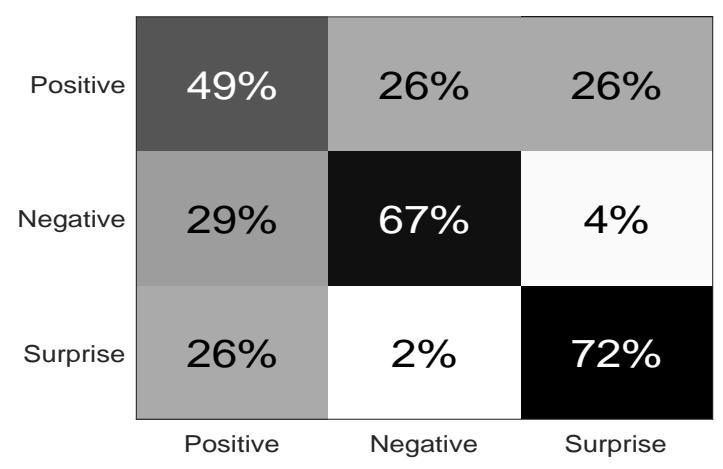

(c) LBP-SIP.

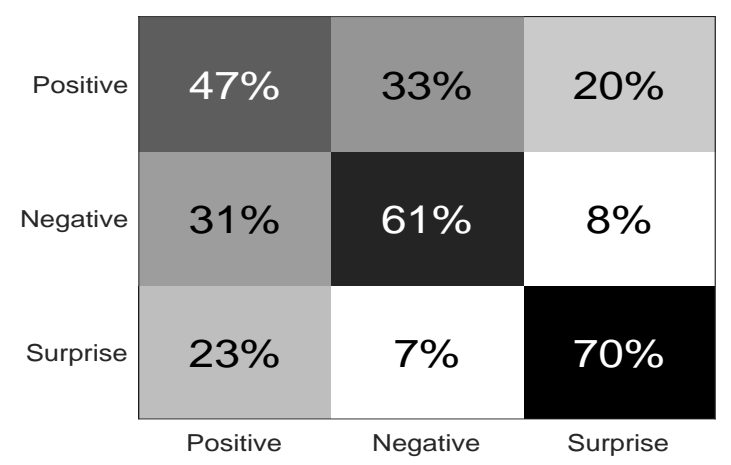

(e) LBP-TOP.

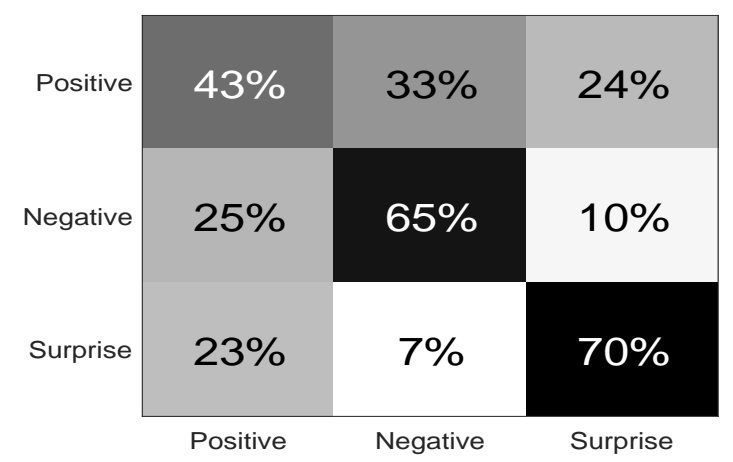

(b) GDLBP-LVP-TOP

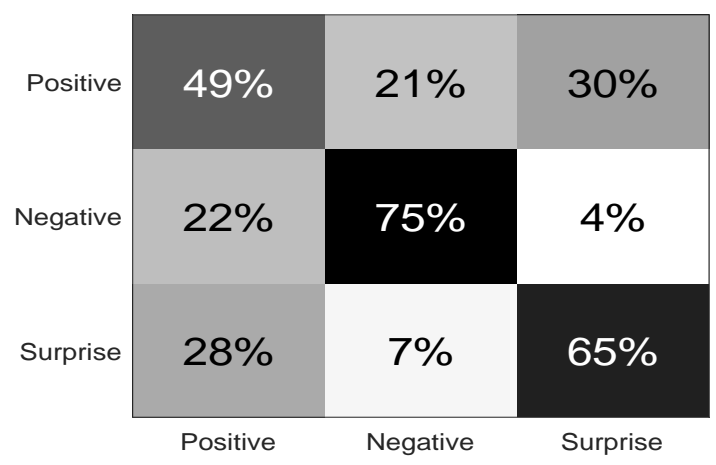

(d) SIP-SDG

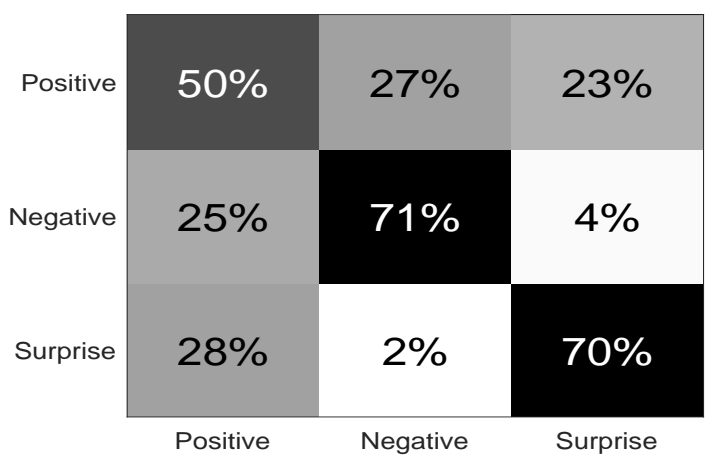

(f) LBP-SDG

Figure 5: The confusion matrices of different features on the SMIC-HS database. 


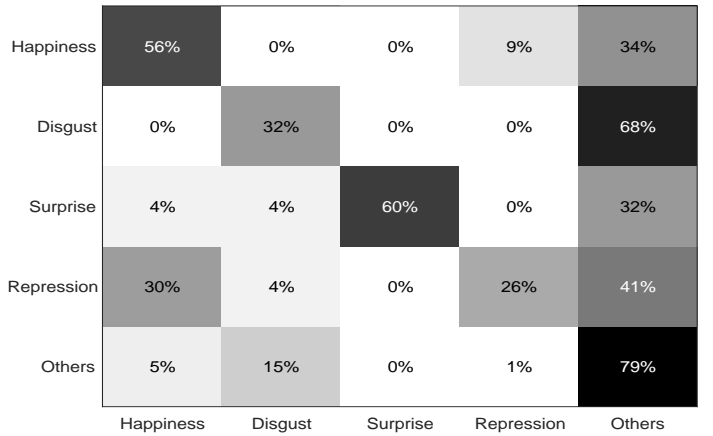

(a) LVP-TOP.

\begin{tabular}{|c|c|c|c|c|c|}
\cline { 2 - 6 } Happiness & $53 \%$ & $3 \%$ & $3 \%$ & $6 \%$ & $34 \%$ \\
\cline { 2 - 6 } Disgust & $3 \%$ & $46 \%$ & $0 \%$ & $0 \%$ & $51 \%$ \\
\hline \multirow{2}{*}{ Repression } & $8 \%$ & $0 \%$ & $60 \%$ & $0 \%$ & $32 \%$ \\
\hline \multirow{2}{*}{ Others } & $5 \%$ & $12 \%$ & $2 \%$ & $2 \%$ & $41 \%$ \\
\cline { 2 - 6 } & $5 \%$ & $4 \%$ & $79 \%$ \\
\hline
\end{tabular}

(c) LBP-SIP

\begin{tabular}{|c|c|c|c|c|c|}
\hline Happiness & $66 \%$ & $6 \%$ & $0 \%$ & $16 \%$ & $13 \%$ \\
\hline Disgust & $8 \%$ & $48 \%$ & $2 \%$ & $0 \%$ & $43 \%$ \\
\hline Surprise & $0 \%$ & $8 \%$ & $60 \%$ & $0 \%$ & $32 \%$ \\
\hline Repression & $30 \%$ & $7 \%$ & $0 \%$ & $48 \%$ & $15 \%$ \\
\hline Others & $6 \%$ & $13 \%$ & $0 \%$ & $2 \%$ & $79 \%$ \\
\hline
\end{tabular}

(e) LBP-TOP.

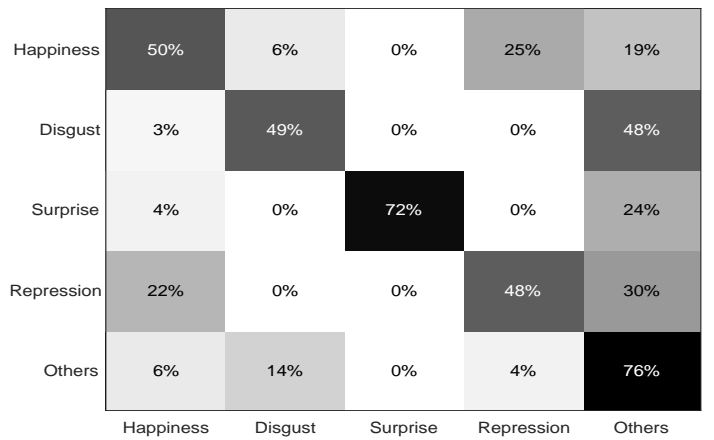

(b) GDLBP-LVP-TOP

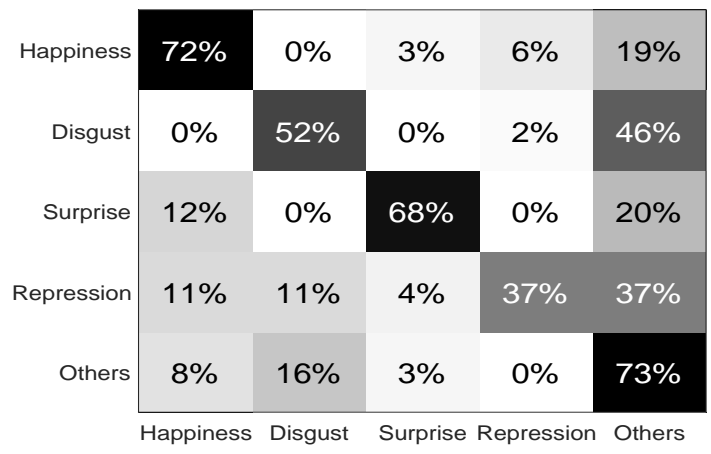

(d) SIP-SDG

\begin{tabular}{|c|c|c|c|c|c|}
\hline Happiness & $66 \%$ & $3 \%$ & $0 \%$ & $16 \%$ & $16 \%$ \\
\hline Disgust & $5 \%$ & $57 \%$ & $0 \%$ & $0 \%$ & $38 \%$ \\
\hline Surprise & $0 \%$ & $8 \%$ & $64 \%$ & $0 \%$ & $28 \%$ \\
\hline Repression & $22 \%$ & $7 \%$ & $0 \%$ & $56 \%$ & $15 \%$ \\
\hline \multirow[t]{2}{*}{ Others } & $7 \%$ & $14 \%$ & $0 \%$ & $3 \%$ & $76 \%$ \\
\hline & Happiness & Disgust & Surprise & Repression & o \\
\hline
\end{tabular}

(f) LBP-SDG.

Figure 6: The confusion matrices of different features on the CASME II database. 